\title{
Transgenerational Rescue of a Genetic Defect in Long-Term Potentiation and Memory Formation by Juvenile Enrichment
}

\author{
Junko A. Arai, ${ }^{1 \star}$ Shaomin Li, ${ }^{1 \star}$ Dean M. Hartley, ${ }^{2}$ and Larry A. Feig ${ }^{1}$ \\ ${ }^{1}$ Sackler School of Biomedical Sciences and Departments of Biochemistry and Neuroscience, Tufts University School of Medicine, Boston, Massachusetts \\ 02111, and ${ }^{2}$ Department of Neurological Sciences, Rush University Medical Center, Chicago, Illinois 60612
}

The idea that qualities acquired from experience can be transmitted to future offspring has long been considered incompatible with current understanding of genetics. However, the recent documentation of non-Mendelian transgenerational inheritance makes such a "Lamarckian"-like phenomenon more plausible. Here, we demonstrate that exposure of 15-d-old mice to 2 weeks of an enriched environment (EE), that includes exposure to novel objects, elevated social interactions and voluntary exercise, enhances long-term potentiation (LTP) not only in these enriched mice but also in their future offspring through early adolescence, even if the offspring never experience EE. In both generations, LTP induction is augmented by a newly appearing cAMP/p38 MAP kinase-dependent signaling cascade. Strikingly, defective LTP and contextual fear conditioning memory normally associated with ras-grf knock-out mice are both masked in the offspring of enriched mutant parents. The transgenerational transmission of this effect occurs from the enriched mother to her offspring during embryogenesis. If a similar phenomenon occurs in humans, the effectiveness of one's memory during adolescence, particularly in those with defective cell signaling mechanisms that control memory, can be influenced by environmental stimulation experienced by one's mother during her youth.

Key words: synaptic plasticity; LTP; kinase; environmental; fear; memory formation

\section{Introduction}

An enriched environment (EE), which can include exposure to stimulatory objects, enhanced social interactions and opportunities for exercise, causes dramatic changes in brain function including enhanced learning and memory (Hebb, 1949; Williams et al., 2001; Meshi et al., 2006), and a delay in the onset (Lazarov et al., 2005; Spires and Hannan, 2005) or even a reversal (Fischer et al., 2007) of neurodegenerative diseases in animals. Many changes in the brain have been observed in response to EE, such as enhanced dendritic branching (Greenough and Volkmar, 1973; Bruel-Jungerman et al., 2005) synaptic density (Rampon et al., 2000), and neurogenesis (Paylor et al., 1992; Kempermann and Gage, 1998; Auvergne et al., 2002; Bruel-Jungerman et al., 2005) (for review, see Nithianantharajah and Hannan, 2006). However, little is known about EE-induced alterations of specific signaling pathways that mediate its effects on neuronal function in either normal or diseased states. We recently showed that 30 -d-old mice, previously exposed to 2 weeks of EE, express a novel signaling cascade that contributes to the induction of long-term potentiation (LTP), a form of synaptic plasticity that is known to be important for learning and mem-

\footnotetext{
Received 0ct. 17, 2008; revised Dec. 14, 2008; accepted Dec. 16, 2008.

This work was supported by National Institutes of Health Grant CA R01CA47391 to L.A.F. and the Tufts Center for Neuroscience Research Electrophysiology and Behavior Core Facilities (P30-NS047243).

*J.A.A. and S.L. contributed equally to this work.

Correspondence should be addressed to Larry A. Feig at the above address. E-mail: larry.feig@tufts.edu.

S. Li's present address: Center for Neurologic Diseases, Brigham and Women's Hospital, 77 Avenue Louis Pasteur, HIM 730, Boston, MA 02115-5817.

DOI:10.1523/JNEUROSCI.5057-08.2009

Copyright $\odot 2009$ Society for Neuroscience $\quad$ 0270-6474/09/291496-07\$15.00/0
}

ory. In particular, EE induces the appearance of an otherwise latent NMDA receptor/cAMP/p38 MAP kinase signaling pathway that adds an additional signaling input into the induction of LTP in the CA1 region of the hippocampus (Li et al., 2006b). This effect of EE is age-dependent, since it does not induce this signaling cascade in adult (6 month old) mice ( $\mathrm{Li}$ et al., 2006b).

Ras-GRF1 (Cen et al., 1992; Shou et al., 1992) and Ras-GRF2 (Fam et al., 1997) constitute a family of calcium/calmodulinregulated exchange factors (Farnsworth et al., 1995) that activate both Ras and Rac GTPases. Ras-GRF2 mediates NMDA receptor activation of Erk Map kinase and LTP in the CA1 hippocampus, while Ras-GRF1 mediates NMDA receptor activation of p38 Map kinase and LTD at this synapse (Li et al., 2006a). ras-grf1 knockout (k/o) mice display normal contextual fear conditioning, but defective contextual discrimination (Giese et al., 2001). Behavior defects associated with the loss of Ras-GRF2 have yet to be documented. We showed previously, that signaling through an EEinduced cAMP/p38/LTP signaling cascade compensates for defective Erk signaling associated with the loss of Ras-GRF2 in 1-month-old mice and restores LTP to normal levels (Li et al., 2006b). Here, we demonstrate that environmental enrichment during youth has dramatic additional powers. It enhances LTP in the future offspring of enriched juvenile mice and masks defective LTP and contextual fear memory in the offspring of enriched juvenile ras-grf knock-out mice.

\section{Materials and Methods}

Mice. ras-grf1(-/-)/ras-grf $2(-/-)$ mice (ras-grf mice) were described previously ( $\mathrm{Li}$ et al., 2006b). These mice were maintained on a mixed 


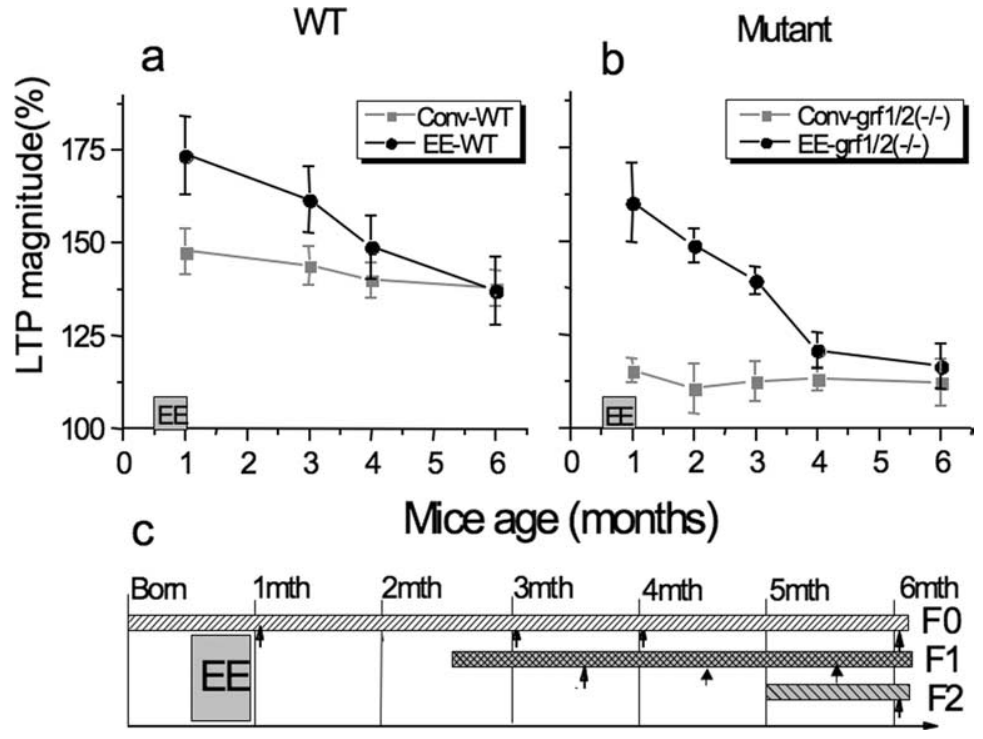

Figure 1. Long-lasting effects of juvenile enrichment. WT (a) or ras-grf knock-out (b) mice (1 month old) were exposed to an enriched environment for 2 weeks and then placed back into conventional environment for various times. LTP was then assayed. Experiments represent mean \pm SEM from at least six brain slices from at least two independent experiments. $c$, A timeline that explains these experiments (F0) and experiments described in Figure 2 (F1), and Figures 2-5 (F1 and F2). The bars represent the life cycle of each generation relative to each other and when animals were tested (arrows).

C57BL/6Jx129 background. Wild-type (WT) mice of the same background were also used.

Hippocampal slice preparation. Mice were killed by halothane inhalation. Then the animals were decapitated and the brain was rapidly removed and submerged in ice-cold oxygenated sucrose-enhanced artificial CSF (ACSF) cutting solution (206 mM sucrose, $2 \mathrm{~mm} \mathrm{KCl,} 2 \mathrm{~mm}$ $\mathrm{MgSO}_{4}, 1.25 \mathrm{~mm} \mathrm{NaH}_{2} \mathrm{PO}_{4}, 1 \mathrm{~mm} \mathrm{CaCl}, 1 \mathrm{~mm} \mathrm{MgCl}_{2}, 26 \mathrm{~mm} \mathrm{NaHCO}_{3}$, $10 \mathrm{~mm}$ D-glucose, $\mathrm{pH} 7.4,315 \mathrm{mOsm}$. Transverse slices $(350 \mu \mathrm{m}$ thickness) from the middle portion of each hippocampus were cut with a vibroslicer. After dissection, slices were incubated in ACSF that contained the following (in mM): $124 \mathrm{NaCl}, 2 \mathrm{KCl}, 2 \mathrm{MgSO}_{4}, 1.25 \mathrm{NaH}_{2} \mathrm{PO}_{4}$, $2 \mathrm{CaCl}_{2}, 26 \mathrm{NaHCO}_{3}, 10$ D-glucose, $\mathrm{pH} 7.4,310 \mathrm{mOsm}$, in which they were allowed to recover for at least $90 \mathrm{~min}$ before recording. A single slice was then transferred to the recording chamber and submerged in a continuous perfusion of ACSF that had been saturated with $95 \% \mathrm{O}_{2}$ and $5 \%$ $\mathrm{CO}_{2}$. Slices were incubated in the recording chamber for $20 \mathrm{~min}$ before stimulation.

Electrophysiological recordings. To record field EPSPs (fEPSPs) in the CA1 region of the hippocampus, standard procedures were used. A unipolar stimulating electrode (World Precision Instruments) was placed in the Schaffer collaterals to deliver test and conditioning stimuli. A borosilicate glass recording electrode filled with ACSF was positioned in stratum radiatum of CA1, 250-350 $\mu \mathrm{m}$ from the stimulating electrode. Test stimuli were applied at low frequency $(0.05 \mathrm{~Hz})$ at a stimulus intensity that elicited a fEPSP amplitude that was $\sim 50 \%$ of maximum, and the test responses were recorded for 20-30 min before beginning the experiment to assure stability of the response. To induce LTP, four theta burst stimulations (TBS) separated by $20 \mathrm{~s}$ were applied to the CA1 area. The TBS consisted of 15 bursts of 4 pulses at $100 \mathrm{~Hz}$, delivered at an interburst interval of $200 \mathrm{~ms}$ were used to induce LTP. The field potentials were amplified $100 \times$ using Axon Instruments $200 \mathrm{~B}$ amplifier and digitized with Digidata 1322A. The data were sampled at $10 \mathrm{kHz}$ and filtered at $2 \mathrm{kHz}$. Traces were obtained by pClamp 9.2 and analyzed using the Clampfit 9.2.

For inhibitor studies, pharmacological agents were added to the perfusion solution 30 min before recordings were initiated. Data analysis was as follows: The fEPSP magnitude was measured using the initial fEPSP slope and three consecutive slopes $(1 \mathrm{~min})$ were averaged and normalized to the mean value recorded $20 \mathrm{~min}$ before conditioning stimulus. Data were pooled across animals of the same treatment and genotype and are presented as mean \pm SEM. Values expressed here represent
60 min time points after conditioning stimulus was initiated, unless stated otherwise. The following statistical analysis was carried out: the same time window samples of the control (cont) and treatment were compared using paired, two-tailed Student's $t$ test. The difference between the effects of different groups was tested by one-way ANOVA. An effect was considered significant if $p<0.05$. Student-Newman-Keuls post hoc tests were used to examine the significance of multiple pairwise comparisons.

Enrichment protocol. Protocols for EE were similar to that used previously (Li et al., 2006b). Where possible, siblings were divided equally between test and control cohorts. Enriched environment included an enriched cage $(45 \times$ $30 \times 25 \mathrm{~cm}$ ) containing plastic play tubes, cardboard boxes, running wheel, various pet toys, and nesting material that were all changed or rearranged every other day to provide novel stimulation. The EE group consisted of 15-dold ras-grf k/o or WT mice that explored the enriched cage for $6 \mathrm{~h}$ per day for $14 \mathrm{~d}$. Agematched control animals were housed three to four per cage in standard cages containing only pine chip bedding.

In a key negative control experiment, mice were handled the same as enriched mice for $14 \mathrm{~d}$, but they were placed into cages identical to their home cage. Under these conditions no significant effect on LTP was observed. In addition, no effect on LTP was observed when 28- to 30-d-old mice were placed in an enriched environment for the first time $2 \mathrm{~h}$ before being killed for LTP assays.

Contextual fear conditioning. The apparatus for fear conditioning consisted of a shock chamber $(9.5 \times 9.5 \times 18$ inches $)$, an electrical shock producer, a photobeam scanner, and a computerized workstation from Motor Monitor system (Hamilton Kinder). The floor of the chamber consisted of 24-bar inescapable shock grids. The walls of the chamber were transparent, thus freezing responses of mice in the chamber could be automatically recorded with the photobeam scanner system. Onemonth-old mice were handled each morning for $5 \mathrm{~d}$ before beginning of experiments. Training sessions were all performed during daylight hours. During the training session, mice were put individually into the chamber and allowed to explore freely the environment for $2.5 \mathrm{~min}$, and during the last $2 \mathrm{~s}$, an electrical footshock $(0.75 \mathrm{~mA})$ was delivered. After the footshock, mice were allowed to stay in the chamber for another 2.5 min before being returned to their home cages. Throughout the procedures, freezing responses were recorded with a $5 \mathrm{~s}$ interval sampling. Freezing response was judged as complete immobility of any part of the body except for respiratory movements. Freezing response during the first $2 \mathrm{~min}$ in the training session was recorded as baseline freezing. Freezing response during the last $2 \mathrm{~min}$ in the training session was recorded as immediate freezing. Retention tests were performed $24 \mathrm{~h}$ after training. During the retention test, each mouse was placed back into the same shock chamber without shock for the measurement of contextual freezing response. Freezing responses were sampled at every $5 \mathrm{~s}$ for duration of $5 \mathrm{~min}$. The floors of all the chambers were cleaned with $75 \%$ ethanol after each test to avoid any possible instinctive odorant cues.

\section{Results}

\section{Long-lasting effects of enriched environment on LTP}

We showed previously that exposing 14-d-old mice to 2 weeks of an enriched environment enhances LTP by unlocking an otherwise latent cAMP and p38 Map kinase-dependent signaling cascade (Li et al., 2006b). To reveal how long this effect lasts, 1 -month-old enriched mice were returned to a conventional environment for various times and then theta-burst-induced LTP 
was assayed. As we and others showed previously (Duffy et al., 2001; Li et al., 2006b), exposure to an enriched environment enhances the magnitude of LTP in the CA1 hippocampus (Fig. 1A) (162 $\pm 9 \%$ of baseline, $n=6$ ). Here, we show that the effect is maintained for $\sim 2$ months, after which it wanes, reaching normal levels after $\sim 3$ months.

We showed previously, and here (Fig. 1B) $(142 \pm 4 \%, n=7)$, that defective LTP associated with the loss of Ras-GRF2 (but not defective LTD associated with the loss of Ras-GRF1) in ras-grf1/2 double knockout mice is enhanced to even a greater degree such that wild-type levels are restored (Li et al., 2006b). Like enhanced LTP observed in wild-type mice described above, this EE-induced rescue of defective LTP in ras-grf knock-out mice also lasts for $\sim 2$ months and then falls back to defective levels found in unenriched knock-out mice after $\sim 3$ months (Fig. $1 B$ ). Figure $1 C$, top bar (F0), shows the timeline for these experiments.

\section{Effects of juvenile enrichment on LTP} are transmitted transgenerationally Because this EE effect on LTP appears to last long enough for these mice to bear offspring, we tested whether it was transmitted transgenerationally. Fifteen-day-old wild-type or ras-grf k/o male and female mice were exposed to EE for $6 \mathrm{~h} / \mathrm{d}$ for $14 \mathrm{~d}$, or to a conventional environment, as described previously (Li et al., 2006b) (see also Materials and Methods). Mice were then allowed to mate. Offspring were raised in a conventional environment for $30 \mathrm{~d}$ and then thetaburst-induced LTP induction at the Schaffer collateral CA1 synapse of the hippocampus of these mice was compared with mice born to nonenriched mothers and to their enriched and nonenriched parents [Fig. 1C, middle bar (F1), for timeline of this experiment].

Figure 2 shows that just like ras-grf1/2 double k/o mice directly exposed to EE (Fig. $2 a$, red circles) ( $145 \pm 5 \%$ of baseline, $n=9$ ), 30 -d-old offspring of these mutant mice also displayed normal LTP levels although they lack Ras-GRF2 (Fig. $2 b$, red circles) (147 $\pm 3 \%$ of baseline, $n=16$ ). As expected, defective LTP was not restored to normal in both nonenriched parents (Fig. $2 a$, black squares) ( $111 \pm 3 \%$ of baseline, $n=10, p<0.001)$ and in their adolescent offspring (Fig. $2 b$, black squares) (116 $\pm 4 \%$ of baseline, $n=9, p<0.001$ ).

A similar phenomenon was observed with wild-type mice (Fig. $2 c, d$ ), where LTP levels were elevated in mice directly exposed to $\mathrm{EE}$ and in their offspring (red circles, parents, $165 \pm 7 \%$ of baseline, $n=9$; offspring, $170 \pm 8 \%$ of baseline, $n=8$ ) compared with nonenriched mice and their offspring (black squares, parents, $147 \pm 6 \%$ of baseline, $n=7$; offspring, $151 \pm 6 \%$ of baseline, $n=9, p<0.05)$. Thus, LTP in adolescent mice is influenced by environmental stimulation experienced by their parents when they were juveniles.

To determine whether the changes in LTP observed in the offspring of enriched wild-type and ras-grf k/o mice were due to the presence of the same new cAMP- and p38-dependent signaling cascade that appears in their parents after EE exposure, the sensitivity of LTP induction in offspring of enriched mice to various inhibitors was evaluated. Figure $3, a$ and $b$, show that in offspring of enriched ras-grf k/o mice, LTP was suppressed by an inhibitor of cAMP (Rp-cAMPs, $119 \pm 5 \%$ of baseline, $n=10$, $p<0.01$ ), or p38 (SB203580, $113 \pm 3 \%$ of baseline, $n=9, p<$ $0.01)$. As we and others have shown previously, these inhibitors did not block LTP in the CA1 hippocampus of 1-month-old WT mice housed conventionally (Zhu et al., 2002; Li et al., 2006a,b). Moreover, in the present study, LTP also was not blocked by an inhibitor of the Erk MAP kinase signaling cascade (Fig. 3b, green triangles) (U0126, $20 \mu \mathrm{M}, 140 \pm 5 \%$ of baseline, $n=7, p>0.05)$, since Erk signaling was already blocked in ras-grf k/o mice. Finally, we found previously that enrichment does not rescue defective LTD that is caused by the loss of Ras-GRF1 in juvenile double ras-grfl/2 (-/-) k/o mice. Here, we show the same result for the offspring of enriched juvenile ras-grf1/2 (-/-) knock-out mice (supplemental Fig. 2, available at www.jneurosci.org as supplemental material).

Similar results were also obtained in inhibitor studies on enhanced LTP observed in offspring of WT enriched mice (Fig. $3 c, d$, vehicle) $(170 \pm 12 \%$ of baseline, $n=9$; SB203580, $142 \pm 4 \%$ of baseline, $n=10, p<0.01$; Rp-cAMPS, $137 \pm 3 \%$ of baseline, $n=$ 


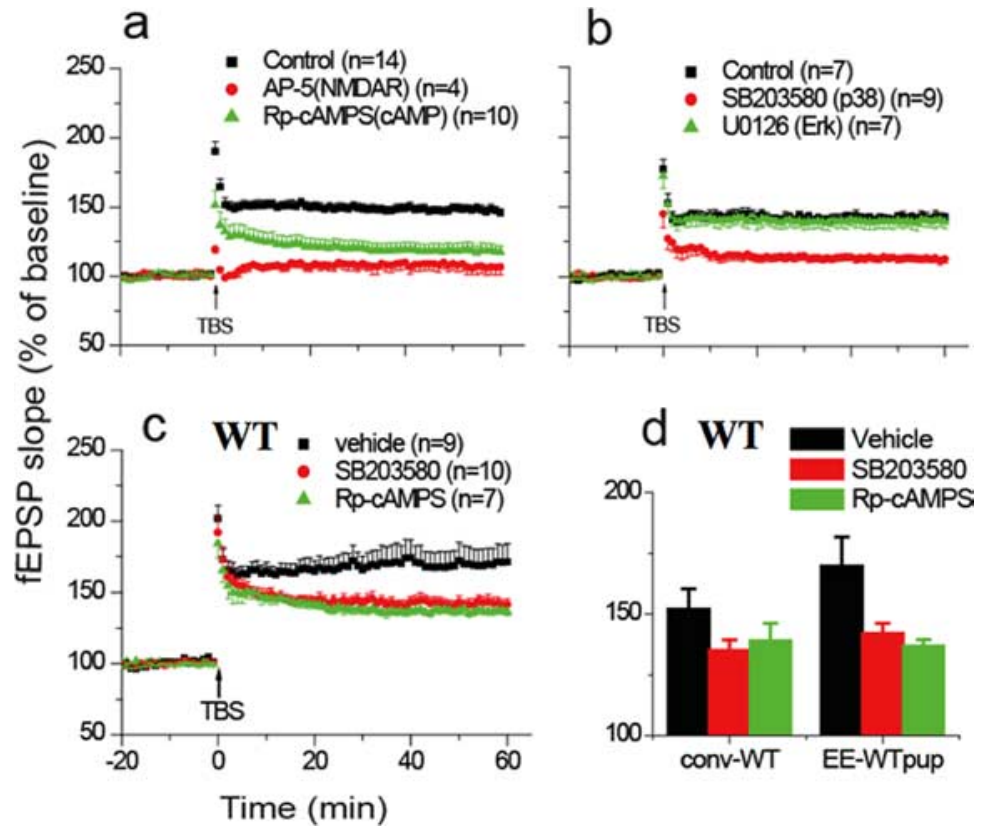

Figure 3. Adolescent offspring of enriched mice display the same novel LTP inducing signaling pathway as their enriched parents. $\boldsymbol{a}$, LTP assays on hippocampal slices from 1-month-old offspring of enriched ras-grf1/grf2 double knock-out mice pretreated with buffer (black squares), NMDA receptor inhibitor (AP-5, $100 \mu \mathrm{m}$ ) (red circles), or cAMP inhibitor (Rp-cAMPS, $100 \mu \mathrm{m}$ ) (green triangles). $\boldsymbol{b}$, Same as in $\boldsymbol{a}$ except that samples were pretreated with vehicle (black squares), p38 inhibitor (SB203580, 5 $\mu \mathrm{m})$, or inhibitor of Erk signaling (U0126, $20 \mu \mathrm{m}$ ) (green triangles). c, Same as in a except that offspring of enriched WT mice were studied and samples were pretreated with vehicle (black squares), p38 inhibitor (red circles), or cAMP inhibitor (green triangles). $\boldsymbol{d}$, Same as in cexcept that offspring of enriched WT mice were studied and data were pooled from multiple experiments. The data represent the average and SEM from the indicated number of samples.

$7, p<0.01)$. Thus, these results support the idea that offspring of enriched juvenile mice inherit the instructions induced by EE that make this newly discovered NMDAR/cAMP/p38/LTP signaling cascade functional even in the absence of EE.

\section{Transgenerational transmission of EE effects on LTP is through the mother before birth}

In experiments described so far, both male- and female-enriched mice were used to breed offspring. To reveal which parent contributes the epigenetic instructions to offspring, enriched mothers were mated with nonenriched mutant fathers and vice versa. We used ras-grf knock-out mice in subsequent experiments because we found no qualitative differences with wild-type mice only quantitative ones in previous experiments. Transgenerational transmission was found to be through the mother because only when she was enriched did her mutant offspring display normal levels of LTP ( $148 \pm 4 \%$ of baseline, $n=13$ vs $116 \pm 3 \%$ of baseline, $n=11, p<0.001$ ) (Fig. $4 a$ ).

We next determined whether transgenerational transmission from the enriched mother to her future offspring occurs before or after their birth. To this end, pups born to ras-grf k/o mothers who were enriched during their youth were immediately placed with nonenriched mutant foster mothers (Fig. $4 b$, EE-Biologic, black squares) ( $147 \pm 6 \%$ of baseline, $n=14)$, who reared them for 1 month in the absence of EE. Alternatively, pups born to mutant mothers that were not enriched during their adolescence were immediately placed with enriched mutant foster mothers (EE-Foster-red circles, $118 \pm 5 \%$ of baseline, $n=14$ ) and reared in the absence of EE for 1 month. Figure $4 b$ shows that mice whose biological mothers were enriched, but were raised by nonenriched mothers, displayed normal levels of LTP despite the absence of Ras-GRF proteins. In contrast, mice born to nonen- riched mothers and reared by enriched foster mothers displayed suppressed levels of LTP consistent with the lack of Ras-GRF proteins. Thus, once the mice are born the enrichment history of the mouse nurturing them does not influence the mechanism of LTP in these mice. These studies show that transgenerational inheritance of the effect of EE occurs before birth.

\section{Transgenerational transmission is limited to F1 generation}

To determine whether this phenomenon is transmitted across multiple generations, $\mathrm{F} 2$, second generation ras-grf $\mathrm{k} / \mathrm{o}$ mice were generated from the offspring of enriched ras-grf k/o mice [Fig. 1C, timeline for experiment, bottom bar (F2)]. Again, these mice were raised continuously in a conventional environment so that they never experienced EE. Figure $4 c$ shows that the effect of EE was almost completely lost by the second generation, since LTP levels (125 $\pm 3 \%$ of baseline, $n=14$ ) were not statistically different from offspring of nonenriched mutant mice $(114 \pm 3 \%$ of baseline, $n=12, p>0.05)$. These findings imply that epigenetic transmission is to somatic cells in the developing offspring. One possible explanation for the limited transmission of this EE effect is that the phenotype ends at an younger age in the offspring of enriched mice than in their parents, such that it is no longer present and thus not transferable to offspring by the time the F1 generation are old enough to reproduce. Figure $4 d$ supports this hypothesis by showing that the magnitude of LTP induction in ras-grf k/o mice directly exposed to EE is the same as in their offspring when they are both 1 month old ( $151 \pm 8 \%$ of baseline, $n=11$ vs $157 \pm$ $9 \%$ of baseline, $n=6, p>0.05)$. However, by 3 months of age, when mice would be pregnant and transmitting the EE effect to their developing offspring, $\sim 80 \%$ of the effect is maintained in mice directly exposed to EE ( $142 \pm 4 \%$ of baseline, $n=6)$, but virtually all of it is lost in their offspring (118 $\pm 4 \%$ of baseline, $n=6)$. Defining why the effect ends when the offspring are younger than their parents will require further experimentation; however, one possibility is that the offspring are exposed to the effects of EE when they are younger (i.e., in utero compared with postnatal days 15-30 for their parents). Thus, if the effects of EE last for similar lengths of time in both cases, it will end at an earlier age in the offspring.

\section{EE effects on contextual memory in ras-grf knock-out mice are transmitted transgenerationally}

To determine whether juvenile enrichment of can rescue a memory defect in the offspring of mice with a genetic defect, contextual fear conditioning experiments were performed on ras-grf knock-out mice. In contextual fear conditioning, an animal is placed in a training cage and a mild footshock is applied, such that the mouse associates the context of the cage with the shock. When the mouse is returned to the cage, contextual memory is measured in the form of increased freezing behavior, a characteristic fear response (LeDoux, 2000). This paradigm is a common tool used to study hippocampal memory formation (Phillips and 
LeDoux, 1992). Moreover, hippocampal LTP is thought to participate in this learning paradigm, and exposure to an enriched environment has been shown to rescue defective contextual fear memories in NMDA receptor knock-out mice (Rampon et al., 2000; Duffy et al., 2001).

First, WT and ras-grf1/2 double knockout mice were compared. Consistent with our previous finding that these mice display defective hippocampal LTP due to the lack of Ras-GRF2 (Li et al., 2006a), grf1/2 $(-/-)$ mice also displayed impaired contextual fear memory (Fig. 5A). In particular, freezing times of WT and knock-out mice were the same before the footshock and immediately after, indicating that the knock-out mice responded normally to this stimulus. However, 24 h later, knockout mice froze $\sim 50 \%$ less than WT mice, indicating a defect in hippocampal memory formation (WT $31.3 \pm 5.35 ; \mathrm{KO}$ $\left.17.1 \pm 3.26, F_{(1,30)}=3.047, p=0.0389\right)$. Similar results were obtained with single ras-grf $2 \mathrm{k} / \mathrm{o}$ mice (data not shown).

Consistent with our previous finding that a 2 week exposure to EE rescues defective LTP in juvenile knock-out mice, EE also restored contextual fear memory to WT levels (Fig. $5 B)(\mathrm{k} / \mathrm{o}, 18.3 \pm 5.35$; EE$\mathrm{k} / \mathrm{o}, 31.0 \pm 3.84, F_{(1,22)}=1.504, p=$ $0.0164)$. Figure $5 C$ shows that offspring of enriched ras-grf knock-out mice raised for 1 month in a conventional environment displayed enhanced contextual fear memory compared with similarly housed 1-month-old offspring of nonenriched ras-grf knock-out parents (cont-k/o, $17.1 \pm$ 2.59; EE$\left.\mathrm{k} / \mathrm{o}, 24.9 \pm 2.46, F_{(1,29)}=1.039, p=0.0429\right)$, although WT levels were not quite reached. Moreover, just as the EE enhancement on LTP in ras-grf knock-out mice dissipated as offspring of enriched mice aged (Fig. $4 D$ ), no significant rescue of defective contextual fear memory was observed at 3 months of age (Fig. $5 D)\left(F_{(1,25)}=\right.$ $1.399, p=0.6954)$. Finally, just as EE effects on LTP were not transmitted to the F2 generation, no enhanced contextual fear memory was observed in these mice (Fig. $5 E)\left(F_{(1,22)}=1.149, p=\right.$ $0.5811)$. Thus, juvenile enrichment can restore, at least in part, a genetically induced memory defect in both mice directly exposed to EE, and more remarkably, in their future offspring through their adolescence, even if the offspring do not experience EE.

\section{Discussion}

This study demonstrates the remarkable power of an animal's environment to modulate the signaling network that promotes LTP in the hippocampus and to improve contextual fear memory formation across generations. In particular, we have dramatically expanded our previous finding that exposing young animals to an EE enhances the magnitude of LTP induction in the CA1 hippocampus (Li et al., 2006b), by showing that it also enhances LTP in their future offspring through adolescence, even if the offspring are not exposed to EE. In both generations, this phenomenon involves the addition of cAMP and p38 MAP kinase dependence to LTP induction. We also demonstrated that juvenile enrichment can rescue defective LTP and improve contex- b

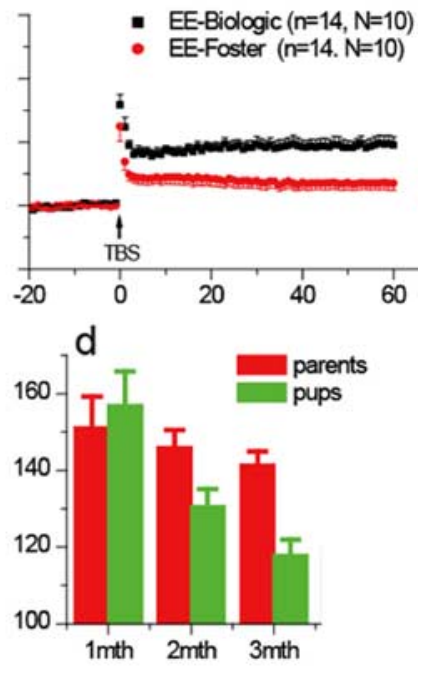

Time (min)

EE-Mom $(n=13, N=9)$

- EE-Father $(n=11, N=8)$ ๘ 1

TBS

neration $(n=14, N=9)$ grf $1 / 2(-/)(n=12, N=8)$
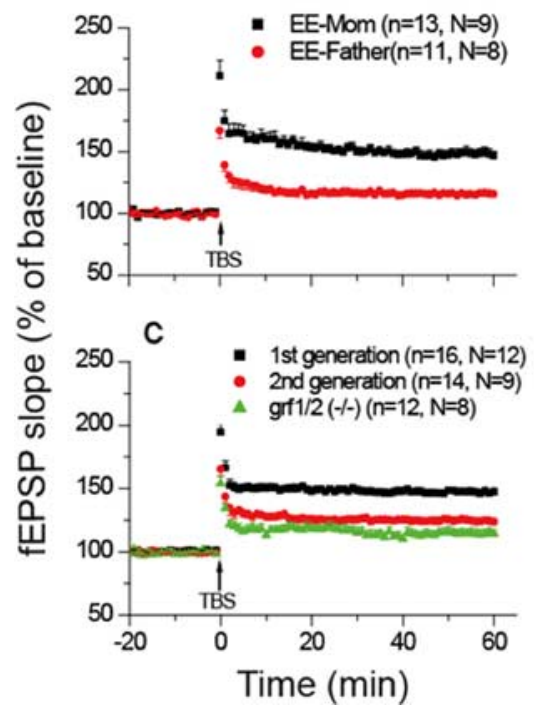

Figure 4. Mechanism of transmission of EE effects across generations. $\boldsymbol{a}$, LTP assays on hippocampal slices from 1-month-old returned to a conventional environment and assayed at 2 months of age and 3 months of age. Data are the average fold increase in fEPSP slopes from three experiments (Fig. 1C).

tual fear memory, normally associated with mutant mice lacking Ras-GRF2 protein, across generations.

Transgenerational epigenetic inheritance is a newly appreciated form of transfer of gene control from one generation to the next. The first example in mammals was detected in "viable yellow" $\left(\mathrm{A}^{\mathrm{VY}}\right)$ and "Axin-fused" (Axin ${ }^{\mathrm{Fu}}$ ) mice where variably penetrant phenotypes are linked to DNA methylation of a retrotransposon driving expression of the $\mathrm{A}{ }^{\mathrm{VY}}$ and Axin ${ }^{\mathrm{Fu}}$ genes (Morgan et al., 1999; Rakyan et al., 2003). This phenomenon can be affected by the environment since maternal diet during pregnancy influences the epigenetic status of $\mathrm{F} 1$ offspring through the alteration of methylation of the $\mathrm{A}^{\mathrm{VY}}$ locus in utero (Waterland and Jirtle, 2003; Dolinoy et al., 2006). Another, more clinically relevant, example is the epigenetic transgenerational effect of endocrine disruptors that alter the methylation state of early germ cells in the developing embryo in utero and generate reproductive disease across multiple generations (Anway and Skinner, 2006).

Multigenerational transmission of epigenetic changes has also been demonstrated to occur through somatic tissues. The bestunderstood example is the transmission of natural variations in maternal behavior, where the degree of licking/grooming behavior of mothers is transmitted to their offspring in the first week of life (Weaver et al., 2004) (for review, see Champagne, 2008). Pups who are exposed to strong nurturing mothers display life-long alterations in the patterns of promoter DNA methylation and changes in expression of specific genes in the hypothalamus that regulate the stress response. These offspring then become strong nurturing mothers and pass the phenotype on to the next generation by their behavior. Interestingly, this effect can be modified by the degree of enrichment to which animals are exposed after weaning (Champagne and Meaney, 2007). 
a
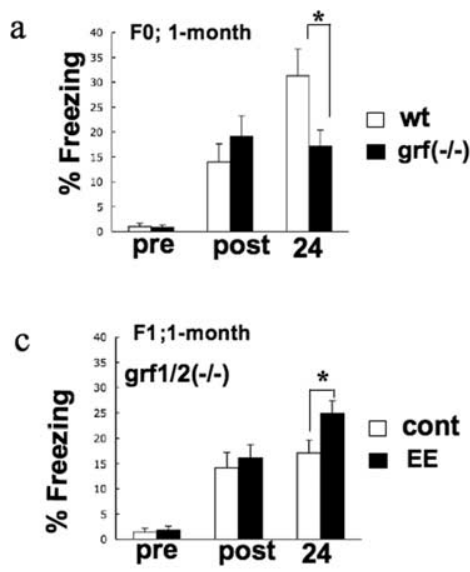

$\mathrm{d}$
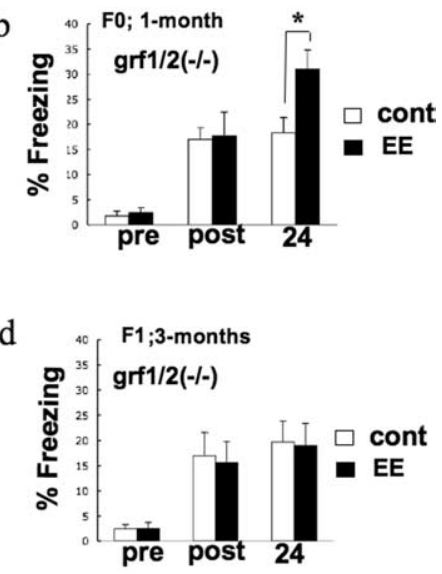

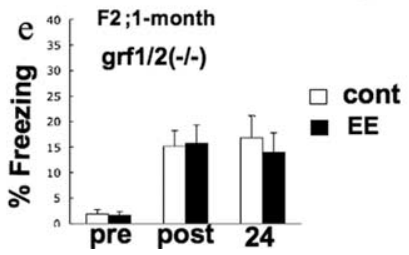

Figure 5. Effect of enrichment on contextual conditioning memory. $\boldsymbol{a}$, ras-grf1/2 double knock-out mice have defective contextual conditioning memory. One-month-old wild-type mice $(\square)(n=17)$ and ras-grf1/2 double knock-out mice $(\square)(n=$ 15) (F0) were subjected to one-trial paradigm training. There was no significant difference in baseline freezing before footshock (pre) or immediately after (post). A retention test was performed $24 \mathrm{~h}$ after training. The data represent the average and SEM from the indicated number of samples. The freezing response in ras-grf1/2 double knock-out mice was significantly lower than that in wild-type mice $(p<0.05$, Mann-Whitney $U$ test). $\boldsymbol{b}$, Enrichment rescues deficient contextual conditioning memory in ras-grf $1 / 2$ double knock-out mice. One-month-old naive ras-grf1/2 double knock-out mice control $(\square)(n=12)$ and enriched ras-grf1/2 double knock-out mice $(\square)(n=12)$ were subjected to one-trial paradigm training as described in $\boldsymbol{a}$. The freezing response in enriched ras-grf1/2 double knock-out mice was significantly higher than that in naive ras-grf1/2 double knock-out mice $(p<$ 0.05 , Mann-Whitney $U$ test). c, Defective contextual conditioning memory is partially rescued in 1-month-old offspring of enriched juvenile ras-grf1/2 double knock-out mice. One-month-old offspring from two litters of either naive ras-grf1/2 double knock-out mice $(\square)(n=15)$ or enriched ras-grf1/2 double knock-out mice $(\square)(n=16)$ were subjected to one-trial paradigm training as in $\boldsymbol{a}$. The freezing response in the offspring of enriched ras-grf1/2 double knock-out mice was significantly higher than that in naive ras-grf1/2 double knock-out mice ( $p<0.05$, Mann-Whitney $U$ test). $\boldsymbol{d}$, Defective contextual fear memory is not rescued in 3-month-old F1 offspring of enriched juvenile ras-grf1/2 knock-out mice. Experiments were performed as in $\boldsymbol{a}$ except that offspring of enriched knock-out mice were reared in a standard environment for 3 months before being tested for fear conditioning response (control, $n=12)(\mathrm{EE}, n=15)$. e, Defective contextual conditioning memory is not rescued in the $\mathrm{F} 2$ offspring of enriched juvenile ras-grf1/2 knock-out mice. Experiments were performed as in $\boldsymbol{a}$ except that 1-month-old F2 offspring of enriched knock-out mice were used (control, $n=11$ ) ( $\mathrm{EE}, n=13$ ).

The phenomenon described here is clearly distinct from the studies described above. For example, epigenetic transmission from mothers to newborn pups through licking/grooming occurs after the birth of affected offspring. In contrast, in our study it occurs before birth because the effect is maintained even if offspring are raised by nonenriched foster mothers.

We also showed that the effect of juvenile enrichment on LTP lasts for $>3$ months, long enough for the effects to be present when the enriched female becomes fertile and pregnant. The idea that the effect of enrichment in the mother can be passed on to offspring during embryogenesis is consistent with a behavior study from $>20$ years ago, which showed that exposure of pregnant rats to an enriched environment enhances the maze learning abilities of their offspring, even if the offspring are raised by nonenriched foster mothers (Kiyono et al., 1985). Our findings may also explain, at least in part, findings from another behavior study from $>20$ years ago, where offspring of female rats exposed to an enriched environment before pregnancy "inherit" their parent's enhanced exploratory behavior and learning skills (Dell and Rose, 1987).

Another distinct feature of our findings is that juvenile enrichment affects LTP in the next generation but not in subsequent ones, possibly because the effects of this $\mathrm{EE}$ wears off faster in the offspring, such that it is not present when they become fertile. Finally, our work is the first to demonstrate the inheritance of a change in a signaling pathway that promotes LTP, and enhancement of contextual memory formation. It is also the first to demonstrate that defects in synaptic plasticity and memory formation caused by a genetic mutation can be reversed, at least in part, in an animal by the environment to which its mother was exposed during her youth.

How juvenile enrichment unlocks an otherwise latent cAMP/p38-dependent signaling cascade to enhance LTP in both enriched mice and in their future offspring remains to be revealed. For example, which component of EE, enhanced social interaction, exposure to novel stimuli or exercise is mostly responsible? Moreover, while it is clear that the EE effect must be indirect in the offspring of enriched mice, possibly through the endocrine system of their mother, is this also true for mice exposed directly to EE? Nevertheless, it is likely that in both generations a longlasting change in the transcription of a rate-limiting gene(s) product that promotes this signaling cascade is involved.

It is highly likely that an enriched environment influences many signaling cascades in the brain and a variety of memory functions in young mice, not just the ones we detected here. Thus, EE likely influences many aspects of animal behavior that could be passed on to the next generation. We could clearly detect EE-induced enhancement of contextual fear memory across generations of ras-grf knock-out mice that correlated tightly with $\mathrm{EE}$ induced enhancement of LTP. However, we found that the 2 week EE paradigm used in these experiments had only a small, if any, effect on contextual fear memory in wild-type mice (data not shown). Thus, we could not confirm that EE effects on this particular behavior could be transmitted transgenerationally in normal mice. This could be a reflection of the fact that the enhancement of contextual fear memory by juvenile enrichment is most important when learning capabilities are suppressed, such as in ras-grf knock-out mice or possibly in learning-disabled humans. Another possibility is that contextual fear memory is not the behavior that is most sensitive to the novel cAMP/p38/LTP pathway that we have identified. Contextual fear memory can be demonstrated in all animal ages, but EE can only activate the cAMP/ p38/LTP pathway in young mice and in their offspring through adolescence. Future experiments are aimed at specifically blocking this newly identified NMDAR/p38/LTP signaling cascade in mice, without blocking other cascades that influence synaptic plasticity. In this way, we hope to reveal its unique contribution to adolescent behavior. The fact that a mechanism has evolved to insure that this signaling cascade is functional through early adolescence in the offspring of enriched mice, even if the offspring 
are not exposed to EE, strongly argues that its role in adolescent behavior is a very important one.

The enriched environment used as an experimental paradigm in these studies may actually be more natural than a conventional laboratory environment that may border on sensory deprivation. Thus, the transgenerational inheritance of this new LTPinducing signaling pathway may be a mechanism that has evolved to protect one's offspring from deleterious effects of sensory deprivation, which may be particularly potent in the young and exacerbated by the presence of mutations in signaling molecules like GRF proteins that contribute to synaptic plasticity. Overall, this study highlights the power of environmental stimulation during youth to influence the composition of signaling networks that influence synaptic plasticity and memory formation, not only in the enriched animal, but also in their future offspring during their youth.

\section{References}

Anway MD, Skinner MK (2006) Epigenetic transgenerational actions of endocrine disruptors. Endocrinology 147:S43-S49.

Auvergne R, Leré C, El Bahh B, Arthaud S, Lespinet V, Rougier A, Le Gal La Salle G (2002) Delayed kindling epileptogenesis and increased neurogenesis in adult rats housed in an enriched environment. Brain Res 954:277-285

Bruel-Jungerman E, Laroche S, Rampon C (2005) New neurons in the dentate gyrus are involved in the expression of enhanced long-term memory following environmental enrichment. Eur J Neurosci 21:513-521.

Cen H, Papageorge AG, Zippel R, Lowy DR, Zhang K (1992) Isolation of multiple mouse cDNAs with coding homology to Saccharomyces cerevisiae CDC25: identification of a region related to Bcr, Vav, Dbl and CDC24. EMBO J 11:4007-4015.

Champagne FA (2008) Epigenetic mechanisms and the transgenerational effects of maternal care. Front Neuroendocrinol 29:386-397.

Champagne FA, Meaney MJ (2007) Transgenerational effects of social environment on variations in maternal care and behavioral response to novelty. Behav Neurosci 121:1353-1363.

Dell PA, Rose FD (1987) Transfer of effects from environmentally enriched and impoverished female rats to future offspring. Physiol Behav 39:187-190.

Dolinoy DC, Weidman JR, Waterland RA, Jirtle RL (2006) Maternal genistein alters coat color and protects Avy mouse offspring from obesity by modifying the fetal epigenome. Environ Health Perspect 114:567-572.

Duffy SN, Craddock KJ, Abel T, Nguyen PV (2001) Environmental enrichment modifies the PKA-dependence of hippocampal LTP and improves hippocampus-dependent memory. Learn Mem 8:26-34.

Fam NP, Fan WT, Wang Z, Zhang LJ, Chen H, Moran MF (1997) Cloning and characterization of Ras-GRF2, a novel guanine nucleotide exchange factor for Ras. Mol Cell Biol 17:1396-1406.

Farnsworth CL, Freshney NW, Rosen LB, Ghosh A, Greenberg ME, Feig LA (1995) Calcium activation of Ras mediated by the neuronal exchange factor Ras-GRF. Nature 376:524-527.

Fischer A, Sananbenesi F, Wang X, Dobbin M, Tsai LH (2007) Recovery of learning and memory is associated with chromatin remodelling. Nature 447:178-182.

Giese KP, Friedman E, Telliez JB, Fedorov NB, Wines M, Feig LA, Silva AJ (2001) Hippocampus-dependent learning and memory is impaired in mice lacking the Ras-guanine-nucleotide releasing factor 1 (Ras-GRF1). Neuropharmacology 41:791-800.

Greenough WT, Volkmar FR (1973) Pattern of dendritic branching in oc- cipital cortex of rats reared in complex environments. Exp Neurol 40:491-504.

Hebb DO (1949) The organization of behavior. New York: Wiley.

Kempermann G, Gage FH (1998) Closer to neurogenesis in adult humans. Nat Med 4:555-557.

Kiyono S, Seo ML, Shibagaki M, Inouye M (1985) Facilitative effects of maternal environmental enrichment on maze learning in rat offspring. Physiol Behav 34:431-435.

Lazarov O, Robinson J, Tang YP, Hairston IS, Korade-Mirnics Z, Lee VM, Hersh LB, Sapolsky RM, Mirnics K, Sisodia SS (2005) Environmental enrichment reduces Abeta levels and amyloid deposition in transgenic mice. Cell 120:701-713.

LeDoux JE (2000) Emotion circuits in the brain. Annu Rev Neurosci 23:155-184.

Li S, Tian X, Hartley DM, Feig LA (2006a) Distinct roles for Ras-guanine nucleotide-releasing factor 1 (Ras-GRF1) and Ras-GRF2 in the induction of long-term potentiation and long-term depression. J Neurosci 26:1721-1729.

Li S, Tian X, Hartley DM, Feig LA (2006b) The environment versus genetics in controlling the contribution of MAP kinases to synaptic plasticity. Curr Biol 16:2303-2313.

Meshi D, Drew MR, Saxe M, Ansorge MS, David D, Santarelli L, Malapani C, Moore H, Hen R (2006) Hippocampal neurogenesis is not required for behavioral effects of environmental enrichment. Nat Neurosci 9:729-731.

Morgan HD, Sutherland HG, Martin DI, Whitelaw E (1999) Epigenetic inheritance at the agouti locus in the mouse. Nat Genet 23:314-318.

Nithianantharajah J, Hannan AJ (2006) Enriched environments, experience-dependent plasticity and disorders of the nervous system. Nat Rev Neurosci 7:697-709.

Paylor R, Morrison SK, Rudy JW, Waltrip LT, Wehner JM (1992) Brief exposure to an enriched environment improves performance on the Morris water task and increases hippocampal cytosolic protein kinase $\mathrm{C}$ activity in young rats. Behav Brain Res 52:49-59.

Phillips RG, LeDoux JE (1992) Differential contribution of amygdala and hippocampus to cued and contextual fear conditioning. Behav Neurosci 106:274-285.

Rakyan VK, Chong S, Champ ME, Cuthbert PC, Morgan HD, Luu KV, Whitelaw E (2003) Transgenerational inheritance of epigenetic states at the murine $\mathrm{Axin}(\mathrm{Fu})$ allele occurs after maternal and paternal transmission. Proc Natl Acad Sci U S A 100:2538-2543.

Rampon C, Tang YP, Goodhouse J, Shimizu E, Kyin M, Tsien JZ (2000) Enrichment induces structural changes and recovery from nonspatial memory deficits in CA1 NMDAR1-knockout mice. Nat Neurosci 3:238-244.

Shou C, Farnsworth CL, Neel BG, Feig LA (1992) Molecular cloning of cDNAs encoding a guanine-nucleotide releasing factor for Ras p21. Nature 358:351-354.

Spires TL, Hannan AJ (2005) Nature, nurture and neurology: geneenvironment interactions in neurodegenerative disease. FEBS Anniversary Prize Lecture delivered on 27 June 2004 at the 29th FEBS Congress in Warsaw. FEBS J 272:2347-2361.

Waterland RA, Jirtle RL (2003) Transposable elements: targets for early nutritional effects on epigenetic gene regulation. Mol Cell Biol 23:5293-5300.

Weaver IC, Cervoni N, Champagne FA, D’Alessio AC, Sharma S, Seckl JR, Dymov S, Szyf M, Meaney MJ (2004) Epigenetic programming by maternal behavior. Nat Neurosci 7:847-854.

Williams BM, Luo Y, Ward C, Redd K, Gibson R, Kuczaj SA, McCoy JG (2001) Environmental enrichment: effects on spatial memory and hippocampal CREB immunoreactivity. Physiol Behav 73:649-658.

Zhu JJ, Qin Y, Zhao M, Van Aelst L, Malinow R (2002) Ras and Rap control AMPA receptor trafficking during synaptic plasticity. Cell 110:443-455. 